\title{
Reducing costs and pollution in cellular networks
}

\author{
Vincenzo Mancuso and Sara Alouf \\ INRIA Sophia Antipolis Méditerranée, France
}

\begin{abstract}
Cellular wireless networks are expected to provide high quality audio and video services while enabling fast and low cost Internet access to mobile users. The need for green cost efficient networks is twofold: $(i)$ reduce the service price, and (ii) preserve the environment. In this work, we discuss the various strategies that help reducing infrastructure costs, power costs, and greenhouse gas (GHG) emissions with no impairments on the quality of network services. These strategies range in a wide area from enhancing the electronics, to developing new energyaware radio access protocols, to deploying enhanced base stations with tunable capacity. To reduce both capital and operational expenditures, and to reduce the GHG footprint, the manufacturers propose new compact installation with lightweight antenna systems, very efficient power amplifiers, efficient hardware and software. The resulting economy can be up to $50 \%$ or more by reducing the electricity bill, sparing the use of air conditioned, and deploying compact sites that would seldom require maintenance. Recent scientific publications confirm that a very high gain could be achieved by optimizing the use of base stations proactively, and huge additional improvements could be obtained by optimizing power save mechanisms by leveraging traffic statistics.
\end{abstract}

keyword - Cellular networks, Green Base station design.

\section{Introduction}

Wireless communications are expected to be the major worldwide cause of energy consumption within few years, with a devastating impact in terms of pollution and energy waste. The high power needed for operating wireless devices causes huge greenhouse gas (GHG) emissions in the atmosphere, while the portion of energy actually traveling on the communication media is one or two orders of magnitude smaller than the energy consumed by the overall system. Jointly with the environmental impact of new wireless devices, e.g., new towers and base station sites, the highly inefficient use of power might turn in a serious menace for the environment. In particular, base stations cause more than $80 \%$ of the operator's power consumption, which makes the design of base stations a key element for determining both the environmental impact of wireless networking and the operational expenditure. However, the availability of next generation wideband and reliable cellular networks (e.g., LTE and HSPA [1]), can help reducing the GHG footprint by reducing the need for traveling and printing documents, as emerged in a recent study by the Australian operator Telstra.

To give an idea of the actual energy and pollution cost induced by a base station, consider that most of the operating base stations continuously consume at least $2 \mathrm{~kW}$, which are mainly generated by means of fossil fuels, and hence produce as much $\mathrm{CO}_{2}$ as few automobiles in their average utilization cycle. New generation base stations reduced their consumption to typically $800 \mathrm{~W}$ for $2 \mathrm{G}$ systems (GSM), and as low as $430 \mathrm{~W}$ for $3 \mathrm{G}$ systems (UMTS). Manufacturers are further targeting to halve the power consumption of their base stations in two years, and to lower the environmental impact by allowing network operators to deploy cheaper and multi-technology sites. Manufacturers particularly focus on new base station design which would reduce capital expenditures (CAPEX) and operational expenditures (OPEX). To achieve this goal, manufacturers and operators point at optimizing the architecture of the site and using renewable energies, improving the power efficiency of the hardware, and deploying smart resource management tools that activate network resources only when needed.

Site design is fundamental for an efficient usage of energy. For instance, flexible and lightweight sites could be better located to provide uniform coverage with no need for high transmission power. Most importantly, site design is responsible for minimizing ownership and management costs, and for reducing the need for energy-hungry air conditioning. Clearly, site design depends on the availability of more efficient, compact, and low-power consuming electronic devices. A substantial reduction of the power needed to operate a base station also significantly helps the deployment of sites totally or partially operated by means of renewable energies, e.g., solar panels, bio-fuels and eolic turbines, as reported, e.g., by Huawei and Ericsson. 
In terms of hardware improvements, manufacturers are replacing existing power amplifiers with new efficient devices using Digital Pre-Distortion (DPD) or envelope tracking for wideband signals. Noticeably, using these efficient power amplifiers allow to deploy new compact sites which can be operated with half power or less (see Section 4).

Eventually, operators are developing new management tools to reduce the amount of devices operating with low or zero load, which has been shown to be almost as expensive as running at full capacity [2], e.g., by switching off unloaded base stations overnight, while avoiding coverage and capacity degradation. Operators like Huawai claim that using radio and computational resources efficiently might easily turn in a $40 \%$ drop in the operational costs.

Recent scientific publications confirm that power save and quality of service enhancements are not conflicting objectives for wireless network design. Power save modes and operational costs optimization have been studied by means of analytical models and simulations. Sleep mode has been proposed as an interesting solution for low loaded transmitters, and it has been analytically shown that optimal sleep periods can be selected as a function of the statistical distribution of the packet arrival over the wireless interface [3,4]. Optimization is carried out in terms of cost reduction subject to quality of service constraints such as the average or maximum tolerable packet delivery time. The results of [3] can be used to design the behavior of an on/off base station during time intervals in which the expected traffic is stationary and data bursts can be modeled as memoryless arrivals. In case of more general traffic patterns, dynamic programming has been used to characterize the optimal sleeping interval at each sleep mode activation epoch [4]. Joint radio resource management strategies have been proposed for the cooperation between GSM and UMTS systems on the same base station. The analytical study in [5] illustrates benefits offered by the cooperation of $2 \mathrm{G}$ and $3 \mathrm{G}$ systems. In particular, inter-technology handover, i.e., from $2 \mathrm{G}$ to $3 \mathrm{G}$ and vice versa, enables a substantial reduction of the system blocking probability at no additional cost, with no need for increasing the capacity of the network. Furthermore, the decision for a user to select one of the available radio systems can be performed subject to congestion control and energy aware mechanisms, with the interesting conclusion that, under low load conditions, the higher gain is obtained by letting all users use one technology only and putting in sleep mode the base station components operating on other technology. Also, standardization bodies like $3 \mathrm{GPP}$ have started to provide the operators with suitable tools to implement power save mechanisms, e.g., the Continuous Packet Connectivity (CPC) for always on customers using high speed data connections [6].

The survey is organized as follows. Section 2, illustrates the green strategies adopted by vendors and operators. Section 3 introduces the architecture of the newest base stations. Section 4 gives insights on the operational costs and energy consumption of a base station. Section 5 concludes the survey.

\section{Green deployment strategies}

In this section, we focus on the strategies that aim at reducing operator's costs and environmental impact due to the base station deployment and operation. We illustrate how green components have been introduced in the design of the core network and base station sites (possibly targeting renewable power sources), in the adoption of efficient hardware, in the responsible use of packaging and energy, and in the introduction of power save features in resource management.

\subsection{Core network rationalization}

A contribution to green networking comes from the simplification of the core network, and from the optimization of the efficiency of soft-switches through which data streams are routed and controlled. For instance, since 2007, the Australian operator Telstra and Ericsson have been developing an efficient, compact and high-capacity mobile softswitch server to simplify the mobile network. The new soft-switch comes with blade technology (i.e., using electronic boards or blades), which is highly modular, and reduces the energy use of the soft-switch by up to $60 \%$ per subscriber. This innovative mobile soft-switch is suitable for both GSM and UMTS networks, and supports up to eight million subscribers with only two equipment shelves. Interestingly, not only the new technology allows the deployment of fewer soft-switches $(75 \%$ less) to satisfy the traffic demand, but also each new mobile soft-switch is easy to upgrade and exhibits a GHG footprint which is as little as $10 \%$ of that of existing servers. Telstra is planning to replace by 2010 the current 18 regional mobile soft-switch servers spread over Australia, by one mobile soft-switch server cluster using blade technology (deployed at two sites for redundancy and dependability issues). This rationalization should reduce equipment floor space by $85 \%$, cut energy use by $75 \%$ and proportionately reduce the GHG footprint. Similarly, Huawei reports that its large capacity mobile soft-switch server uses a platform, named ATAE, that enables a pool technique with results similar to the Telstra case, and has brought an overall cost reduction of $80 \%$ for the Sichuan mobile network in China. 


\section{$2.2 \quad$ Green base station sites}

Here we show three different examples of site design, each targeting — for different environments - low GHG footprint and low installation, operational, and maintenance costs. Then we discuss the utilization of renewable energy.

\section{Optimized design}

The Tower tube. The target of Ericsson's introduction of a new radio base station site concept, Tower Tube, is threefold: reducing pollution causes, making the wireless network more cost-efficient, and allowing the integration of the site with the surrounding environment. Tower Tube has a modern design for a 5 m-diameter, $40 \mathrm{~m}$-high flexible concrete tower encapsulating all radio base station equipment and antennas. The concrete itself has a lower environmental impact than traditional steel, producing $30 \%$ less $\mathrm{CO}_{2}$ emissions during production and transportation. Unlike commonly adopted sites, the network equipment is installed at the bottom of the site, and then rised by means of an elavator to the top of the tower, next to the antennas. The advantages of this choice are particularly evident in the reduced loss in the feeder between the radio-frequency unit and the antenna, and in the possibility to exploit the wind as a passive cooling system. Energy saving can be quantified in a 40\% reduction of operational costs. Additionally, since Tower Tube occupies 60-75\% less space than conventional sites, site acquisition is cheaper, which means, lower capital expenditures are required. Even lower operational costs are possible with the wind-powered version of the Tower Tube, endowed with five-meter blades and a vertical rotor.

The Capsule Site. Finding new locations for radio base stations is challenging, especially in urban and suburban areas, where obtaining building permit for a base station might require the design of an unobtrusive site, or also the integration of the site as a new landmark in the surroundings. The Ericsson Capsule Site is an example of base station site design for easy deployment in urban areas. The Capsule Site is an all-in-one solution encapsulating all necessary equipments. The site cover is made of composite, weighs $800 \mathrm{~kg}$, and can be delivered and installed in less than a day.

The Flexi base station. An example of efficient site design and management is the Flexi base station by Nokia Siemens Networks. The benefits are as follows: $(i)$ reduce site energy costs up to $70 \%$; $(i i)$ reduce size and weight of network equipments by 80\%; ( $i i i$ ) allow flexible location, i.e., indoor and outdoor, with no need for air conditioning; $(i v)$ shorten antenna feeders; $(v)$ make new sites ready for future radio technologies. The Flexi base station GHG footprint is further diminished by its software-based capacity and capability upgrades. In fact, Flexi base stations can be remotely managed, in terms of tuning the running capacity (i.e., the number of GSM frequencies in use), and also remotely upgraded, canceling the need for site visiting. Flexi base station is also ready for using renewable energy such as solar or wind power.

\section{Renewable power sources}

Considering the demand for new base station installations in many regions where an efficient power grid is not yet available (or where a power grid does not exist at all), the possibility to operate wireless base stations relies on the use of local power generators. In the past, power generators have been using diesel engines, while the current trend is towards the replacement of diesel with bio-fuels, and traditional generators with solar- or wind-powered generators. Unfortunately, a complete replacement is not possible in short terms, since many old installations require way too much energy to be reliably supplied by means of renewable sources. Hence, a consumption reduction strategy is strictly necessary to enable the deployment of renewable energy-powered base stations.

In 2008, GSMA (the association gathering nearly 800 worldwide mobile operators) has launched a plan for deploying renewable energy sources for 118,000 new and existing base stations in developing countries by 2012. The expected green gain is to save 2.5 billion liters of diesel, and cut $\mathrm{CO}_{2}$ emission up to 6.3 million tons per year. Solar energy solutions have been proposed since 2000, at least to operate in conjunction with diesel generators. Ericsson and Alcatel-Lucent, amongst other companies, have proposed solar-powered base stations for macro-coverage and low capacity, useful for rural or low-dense areas.

Although the $100 \%$-solar-powered solution is in the target, and operators like Orange Guinea targeted at having more than one thousand $100 \%$-solar-powered base stations by the end of 2009, many installations are still hybrid, using solar panels and batteries to replace only one out of the two diesel generators usually deployed. Nevertheless, the gain is high, since a typical site consumes approximately 20,000 liters of diesel per year, which can be effectively halved by means of solar panels and batteries. Further gain can be obtained by combining multiple renewable power sources in the same site. By using solar and wind power, plus an auxiliary diesel generator, African operator Zain has deployed a site in Dertu, a remote village in Kenya. The operational cost due to electricity has been reduced by $80 \%$ for that site, compared with using diesel generators only. 


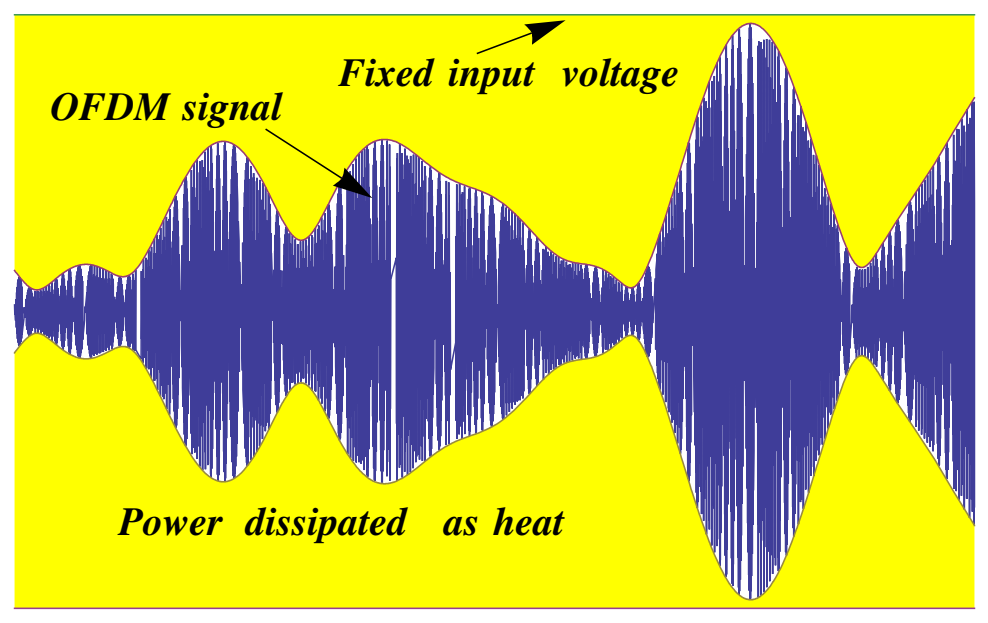

Figure 1: 1024-subcarrier OFDM signal amplified with constant input voltage supply. The power dissipated as heat is huge due to the difference between the signal envelope and the voltage supply level.

\subsection{Energy efficient power amplifiers}

Next generation cellular networks will make a large use of non-constant envelope signals. In particular, the widely accepted adoption of OFDMA systems will challenge the manufacturers to design more efficient and accurate wideband OFDM modulators. In an $N$-subcarrier OFDM, the transmit power is evenly spread over the subcarriers, and the peak-to-average power ratio, i.e., the ratio between the maximum instantaneous power and the average power is equal to $N$ [7]. Thereby, the maximum voltage amplitude at the power amplifier is $\sqrt{N}$ times the average voltage amplitude and it is not convenient to amplify the OFDM signal using a constant input voltage, which would turn into constant power consumption even when the signal is very low (and the unused power is dissipated as heat, cf. Figure 1).

In order to be power-efficient, the average input power at the power amplifier has to be kept as close as possible to the power needed for transmitting the signal. Possible solutions consist in either distort the signal or dynamically adapt the input voltage of the amplifiers.

\section{DPD and Doherty power amplifiers}

Digital Pre-Distortion (DPD) and Doherty techniques are used to adapt the signal to the amplifier's characteristics and to boost-up the power emission when the signal level is above fixed a threshold, thus avoiding excessive signal clipping.

The DPD and linearization technique uses pre-distortion of the signal to be transmitted and operates a compensation for nonlinearities in the final radio-frequency output stage of the amplifier. In the process, it also improves adjacent channels interference and reduces the error vector magnitude - i.e., it improves the modulation accuracyand allows some compensation for the distortion caused by nonlinearities near compression. This technology obtains the best result when DPD and linearization are used as part of a feedback system architecture incorporating active sampling of the output signal. This way, in fact, the system fully compensates for changes in amplifier characteristics with time, temperature, and signal characteristics [8].

The Doherty power amplifier configuration has been originally proposed in 1936 [9]; it uses two amplifying devices driven in parallel, with their outputs combined. One carrier amplifier, operating in Class A/B, provides all the output power until the power required causes it to enter its nonlinear region. A peaking amplifier, operating in Class C, provides additional power when the carrier enters its nonlinear region. While several academic papers have quoted impressively high efficiency capabilities for Doherty amplifiers [8], in practice, at the frequencies and with the high output power which are used in cellular networks, the typical efficiency achieved is around 25\% to 30\% [10]. Doherty amplifiers have a limited bandwidth due to the complicated and essentially narrow-band matching required between the two internal amplifiers. However, the bandwidth available (few $\mathrm{MHz}$ ) is adequate enough for cellular systems.

Huawei, the Chinese telecom equipment provider, has launched a low-power base station adopting DPD and Doherty technologies. In terms of energy, this base station can be run with as low as $500 \mathrm{~W}$, thereby helping operators to save up to $5700 \mathrm{kWh}$ per year, or, equivalently, reduce the $\mathrm{CO}_{2}$ emissions due to energy generation by 1.7 tons of coal per year. Such a low-power demanding hardware can easily rely on renewable power supplies. Huawei's solution has been utilized over 100,000 GSM base stations from 2007, so helping to save about 570 GWh per year, representing 


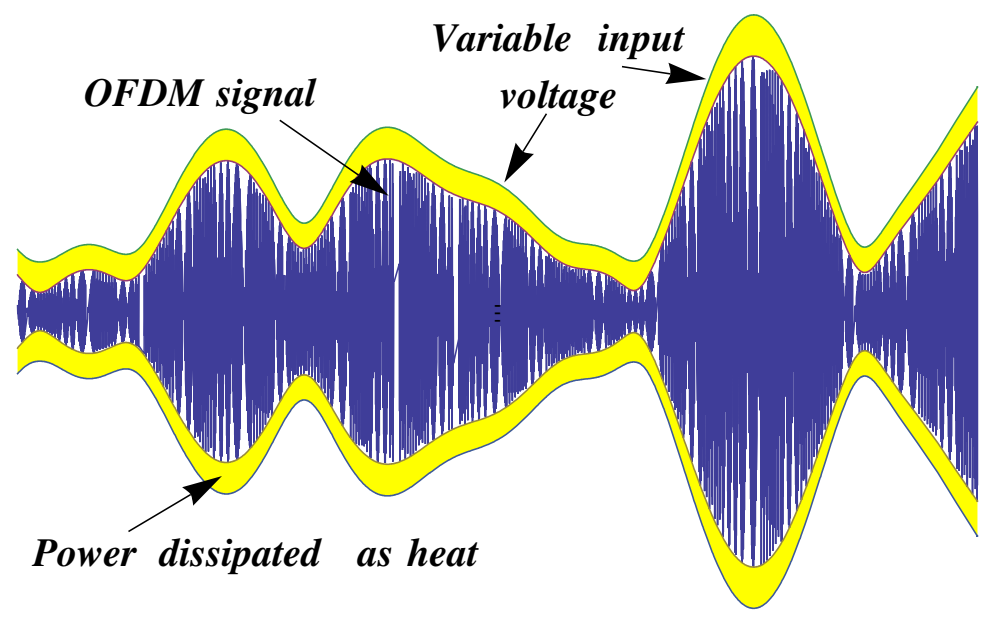

Figure 2: Power amplifiers input voltage can be made variable when envelope tracking is used. The figure shows the case of OFDM signals with 1024 subcarriers: very few power is wasted as heat as the difference between the signal envelope and the voltage supply level is small.

a reduction of $\mathrm{CO}_{2}$ emissions equivalent to burning 170,000 tons of coal.

2. Envelope tracking technology

Envelope tracking has been proposed by Bell Labs researchers in 1937, but it has been efficiently implemented only recently after very fast, low-noise power transistors have became available (using LDMOS, GaN, GaAs, etc.). The basic idea of envelope tracking is as follows: instead of changing the signal to match the power amplifier characteristics, dynamically try to adjust the supply voltage of the power amplifier to match the envelope of the signal to be amplified. Thus, envelope tracking ensures that the output device remains in its most efficient operating region, i.e., in saturation. Figure 1 shows the inefficiency of using power amplifiers with constant input voltage supply. Without envelope tracking, the difference between the constant power fed into the radio-frequency amplifier and the radio-frequency output waveform is dissipated by the power transistor as heat. With envelope tracking, as depicted in Figure 2, the supply voltage tracks the signal envelope, so that the input power closely matches the radio-frequency output power. This matching turns into a dramatic reduction of the energy dissipated as heat. Performing envelope tracking requires a CPU cost that can consume as much as few Watts, which are negligible in comparison to the energy saved (tens or hundreds of Watts), in case of high power amplifiers.

The first practical commercial implementation of envelope tracking is very recent (Nujira, 2008), and can make a significant contribution to the power efficiency of the power amplifiers, improving this from the $15 \%$ of Class A/B power amplifiers to $45 \%$ nowadays, and, according to vendors, the power efficiency will soon reach $60 \%$ using the latest gallium nitride power transistors for radio-frequency. Nujira introduced - and patented-HAT (High Accuracy Tracking), an ultra-high efficient wideband modulator for radio-frequency with power amplifiers that make use of envelope tracking. It is, thereby, suitable for a broad class of non-constant envelope modulators such as those required for OFDM transmissions. HAT modulators for 3G (CDMA), HSPA, DVB, WiMAX and LTE provide the wireless manufacturer with the possibility to design smaller and cost effective base stations which also have a reduced environmental footprint: the first commercial HAT-based radio-frequency unit for WCDMA/HSPA/LTE (Feb. 2009) uses 50\% less energy than traditional devices. By deploying base stations using the HAT modulator, a cellular network with 20,000 base stations could save $35 \mathrm{MW}$, thus saving the operator each year $\$ 37$ million in energy costs, which potentially reduces $\mathrm{CO}_{2}$ emissions by 130,000 tons. Table 1 compares Class A/B, Doherty, and HAT power amplifiers in terms of per-amplifier efficiency and per-network costs. Doherty amplifiers performance are in between traditional Class A/B and HAT performance.

\subsection{Energy management tools}

Energy efficient solutions should be able to correlate the energy consumption and the traffic intensity. As a first step in this direction, wireless operators have proposed the adoption of smart software features for enabling power save at the base station. Here, we introduce some example of such software.

NectAct SQM. Energy conservation can be obtained through traffic-load-driven capacity management at the 
Table 1: efficiency, costs and environmental impact of a 20,000-base station network with different power amplifier technologies.

\begin{tabular}{|c||c|c|c|}
\hline & $\begin{array}{c}\text { Traditional } \\
\text { technology }\end{array}$ & $\begin{array}{c}\text { Doherty } \\
\text { technology }\end{array}$ & $\begin{array}{c}\text { Envelope } \\
\text { tracking }(\text { HAT) }\end{array}$ \\
\hline \hline Power ampl. effic. & $15 \%$ & $25 \%$ & $45 \%$ \\
\hline Power consumption & $51.7 \mathrm{MW}$ & $27.2 \mathrm{MW}$ & $16.1 \mathrm{MW}$ \\
\hline Power cost & $\$ 54.3 \mathrm{M}$ & $\$ 28.6 \mathrm{M}$ & $\$ 17.0 \mathrm{M}$ \\
\hline $\mathrm{CO}_{2}$ emission & 194,600 tons & 102,400 tons & 60,800 tons \\
\hline
\end{tabular}

base stations. Nokia Siemens Networks' NetAct Service Quality Manager (SQM) is a software solution for managing available capacity when traffic load is low. NetAct controls base station power consumption automatically based on pre-configured settings, e.g., NetAct's power save function allows service providers to manage power consumption during times of high and low traffic. Traffic patterns can be managed from network down to individual base station level. A software like NetAct SQM works for $2 \mathrm{G}$ and $3 \mathrm{G}$ systems, and allows to save power by tuning the setting of the base station automatically. For instance, system capacity tuning is scheduled every few tens of minutes, and the actual capacity is chosen as a function of the current traffic load, the history of the traffic load, and the estimate for the traffic load during the next hour. Capacity adjustments consist in changing, e.g., the number of active frequencies for GSM access networks, which can also result in turning off all radios if the traffic during the next hour is expected to fall below a minimum threshold. In particular, the commercial base station software NetAct SQM comes with a strategy named base station power saving at night, which allows the operator to set a time window during which the base station capacity can be reduced. The actual capacity decision is taken in accordance with a traffic profile that is estimated over the hours of the week, and only after a series of preconditions is met: $(i)$ current traffic is below a tunable threshold; $(i i)$ current traffic is within its expected profile (so that traffic estimates can be assumed to not exceed significantly the expected value); (iii) the traffic expectation for the next hour is below the threshold; and ( $i v$ ) no relevant alarms and events are scheduled during the next hour.

Smart Power Management. Another software for the dynamic activation of network equipments is the Smart Power Management (SPM) developed by Nortel. SPM software enables 2G network operators to switch off the radio network equipment when there is no traffic being processed by the system. Nortel estimates the potential power save in up to $33 \%$ reduction in base station power consumption.

Dynamic Power Save. In February 2009, Alcatel-Lucent launched a new feature called Dynamic Power Save, which promises a power consumption reduction by up to $30 \%$ through the possibility to turn off the power amplifiers in GSM transceivers on the basis of the traffic activity monitored by the base station. Interestingly, the company says the software upgrade can be installed on the (roughly) 500,000 Alcatel-Lucent base stations deployed since ten years.

Standby mode. Standby operational modes have been launched since 2007 by Ericsson, whose power save estimate is about $10 \%$ to $20 \%$, depending on the traffic pattern.

\subsection{Best practices for a low-carbon economy}

Remarkable energy economy and GHG reduction can be obtained by adopting responsible practices for the setup and management of the network, beginning with the packaging. A clear example of such green practices can be observed in the green action plan defined by China Mobile - the Chinese cellular operator - for the management of packaging. This strategy is defined by the following six $R$ 's: Recovery $\&$ Recycle refer to using recyclable packaging materials that require low energy cost during their life-cycle. Right $\&$ Reduce refer to using small and lightweight carton design, thereby reducing packaging and transportation costs. Returnable $\&$ Reuse refer to the use of efficient recycling systems in order to extend the life-cycle of packaging materials. Following these directives, the Chinese manufacturer Huawei reported an annual cost reduction of 12,000 $\mathrm{m}^{3}$ of lumber, 2,700,000 liters of oil, $0.75 \mathrm{GWh}$ of energy, and a cut of $6,172,000$ tons in $\mathrm{CO}_{2}$ emissions.

The green strategies introduced so far represent the tools that network operators can leverage to enable low-cost virtual infrastructure and services, contributing to reducing the need for traveling and so to the rising of a low-carbon economy. Since 2007, green technologies by Ericsson, Nokia Siemens Networks, Alcatel-Lucent, Huawei, Nujira, and other leading companies have been used worldwide to reduce energy costs and correspondingly reduce GHG emissions due to power generation. As an example, in 2008, Ericsson set its first carbon footprint target, aiming for a $40 \%$ reduction over five years, and starting with a $10 \%$ reduction in 2009. 
Table 2 summarizes the impact of the different strategies presented so far. Next, we focus on the site cost, illustrate the various cost components incurred when operating a base station, and the potential for the reduction of each of these costs.

Table 2: impact of different strategies, with respect to old-design base stations

\begin{tabular}{|c||c|c|c|c|}
\hline Element & Solution & CAPEX saving & OPEX saving & less CO $\mathrm{CO}_{2}$ \\
\hline \hline Core network & compact soft-switches & $75 \%$ & $90 \%$ & $80 \%-90 \%$ \\
\hline Base station site & radio equipment next to the antenna & $>50 \%$ & $40 \%-70 \%$ & $30 \%$ \\
\hline Power amplifiers & envelope tracking & - & $>50 \%$ & $>50 \%$ \\
\hline Software & site power save & - & up to $40 \%$ & up to $40 \%$ \\
\hline Low-carbon practices & packaging, recycling, bio-fuels & - & $>40 \%$ & $>50 \%$ \\
\hline \hline & Overall impact & $\sim 70 \%$ & $>50 \%$ & $>50 \%$ \\
\hline
\end{tabular}

\section{Architecture of a green base station}

The analysis of expenditures on 3G network site construction shows that base station's infrastructure costs sum up to $30 \%$ of the total cost. Other expenses such as the site acquisition depend on the base station architecture. Smaller size and more power efficient base stations imply reduced investments and operational costs. In order to move further with the cost reduction, operators are asking for flexible base stations, where multiple technologies could be run simultaneously, multiple carriers could be operated on demand, and easy upgrades could be performed by simply updating software components.

From the operator's perspective, running multiple radio technologies in a single base station (multimode base station) means shared capital expenditures for the site and reduced management expenses. As will be detailed in Section 4, a base station mainly consists of the base-band unit, the radio-frequency unit, the transport unit, and a control unit, the other units being auxiliary to these networking units. To make the installation simpler, more flexible, and multimode, Huawei, Alcatel-Lucent and other manufacturers have proposed to locate solely the radio-frequency units on site, and to use the open Common Public Radio Interfaces (CPRI) to communicate with the base-band unit and other units like the remote control and transport units. The base-band unit can be made as small as a $2 U$-unit (i.e., with a $19^{\prime \prime} \times 3.5^{\prime \prime}$ front panel) and can be conveniently installed in existing cabinets such as auxiliary power supply cabinet, transmission equipment cabinet, or other remote equipment cabinets. The radio-frequency unit can be made light enough to be installed on a tower, a pole, or simply against a wall. Thereby, since the radio-frequency unit installation is made easy, the conventional feeder is no longer used, which enables a closer antenna interface and avoids a $3 \mathrm{~dB}$ power loss. This base station can be used for both $2 \mathrm{G}$ and $3 \mathrm{G}$ radio access networks, as in the case of the Flexi base station. In particular, the control unit can be shared between $2 \mathrm{G}$ and $3 \mathrm{G}$ radio-frequency units by simply loading the appropriate software. For example, the previously mentioned Flexi base station was originally meant for GSM/EDGE networks, but it has been upgraded to support all 3GPP technologies from WCDMA/HSPA to LTE, having them all running concurrently in a single unit (Flexi Multiradio base station, on the market since early 2009). This upgrade has been possible just by updating the base station software. Another multimode solution has been recently proposed by the Chinese operator ZTE, whose Unified Hardware Platform supports GSM, CDMA, UMTS, TD-SCDMA, and LTE. Other multi-carrier radio-frequency units are available for CDMA, in which one unit can support several carriers (four to eight carriers, in current implementations), and multiple carriers can share the same antenna system without the need of using a signal combiner.

Finally, it is worth mentioning that manufacturers are developing new software defined radio aimed at easily configuring the radio interface and upgrading the transmission technology at zero cost. For example, ZTE has launched in 2009 its software defined radio solution, to be used for GSM/CDMA/UMTS base stations, and that is also ready to evolve towards LTE.

\section{Base station operational cost analysis}

In this section, we provide the reader with a detailed breakdown of the costs related to the operation of a base station, and in particular we analyze the operational costs due to power consumption. 


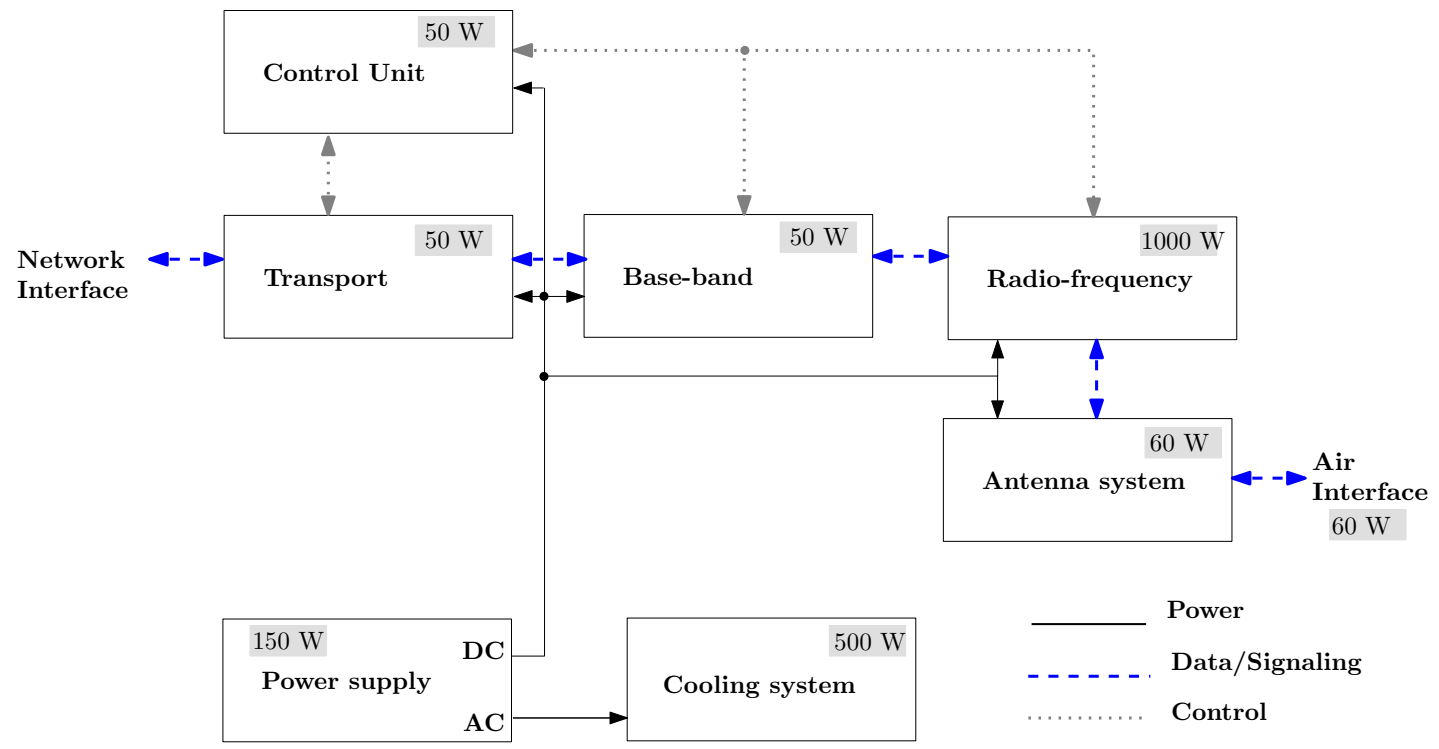

Figure 3: Modular composition of a high power base station. A dual power supply, AC and DC, is usually needed to feed the cooling system and the electronic devices, respectively. Only a minor fraction (e.g., $60 \mathrm{~W}$ ) of the total power consumption (e.g., $\sim 2 \mathrm{~kW}$ ) is radiated on the air.

\subsection{Operational costs}

A base station is a modular composition of several units. The resulting base station building blocks are depicted in Figure 3, and introduced in what follows.

The antenna system includes one or more antennas for one or more radio-frequency bands in use. The base-band process unit is in charge of handling the data and voice streams, by operating digital signal processing and generating and parsing control signals. The radio-frequency unit is the radio-frequency modem that connects the base-band unit to the antenna system, and includes a digital processing board and the power amplifiers necessary to drive the antenna radiation. The control unit provides configuration tools and management interfaces to the base station equipment, and handles alarms and operational logs. The transport unit provides transport services for the data to be exchanged between the base station and the core network, including user generated data, voice and video streams, and control messages, e.g., for handling handover procedures within the radio access network. The cooling system is responsible for the control of the ambient temperature, so that electronic devices would be dependably operated. The power supply unit has to provide the base station equipment with continuous and stable electrical power, and can include power generators, e.g., solar panels and/or diesel generators, and a voltage rectifier for AC/DC conversion.

Note that a base station might include one or more radio-frequency units for different bands, for different $2 \mathrm{G} / 3 \mathrm{G}$ technologies, and for multiple coverage sectors. Also, base-band units could be shared between multiple base stations, thus making it possible to deploy simple base stations which consist of radio-frequency unit, antenna system and power supply only.

Base station installation and upgrade expenses include the acquisition of equipment, the transport to the site, plus the costs for the site property (or rental) and for building the site itself, including towers for the antenna system and water-proof cases for the equipment. These capital expenditures are strongly dependent on the size and the weight of the base station, and can range from a few thousand dollars to a few hundred thousand dollars. However, base station costs are dominated by the operational cost of the energy needed to operate the site. For both $2 \mathrm{G}$ and $3 \mathrm{G}$ base stations, vendors report typical consumption values in the order of several hundreds of Watts $(0.5 \mathrm{~kW}$ to more than $2 \mathrm{~kW}$ ). In particular, base-band, control and transport units operate with very low power, as they require only a few tens of Watts, resulting in as few as $5 \%$ of the total power consumption. The radio-frequency unit consumption in mainly due to the power amplifiers that have to feed the antenna system. This consumption is of the order of several hundreds of Watts, with less than 100 Watts being radiated by the antenna. Noticeably, about half of the 
Table 3: operational costs repartition for a traditional base station provided with a single radio board, a cooling system and an AC/DC rectifier, plus control and transport devices. Original costs are compared with possible cost saving due to technology enhancements and usage of renewable energy.

\begin{tabular}{|c|c|c|c|}
\hline & $\begin{array}{c}\text { Cost impact with } \\
\text { traditional base stations }\end{array}$ & $\begin{array}{l}\text { Relative saving estimate with } \\
\text { technological enhancements }\end{array}$ & $\begin{array}{l}\text { Absolute saving estimate with } \\
\text { technological enhancements }\end{array}$ \\
\hline Control and transport & $\leq 5 \%$ & $\leq 60 \%$ & $\leq 3 \%$ \\
\hline Digital processing (base-band + radio-freq.) & $\leq 10 \%$ & $\leq 50 \%$ & $\leq 5 \%$ \\
\hline Power amplifiers (radio-freq.) & $30 \%-60 \%$ & $\sim 40 \%$ & $\sim 15 \%$ \\
\hline Antenna system & $15 \%-20 \%$ & $\sim 50 \%$ & $\sim 10 \%$ \\
\hline Air conditioning & $10 \%-25 \%$ & $100 \%$ & $10 \%-25 \%$ \\
\hline$A C / D C$ & $\sim 10 \%$ & $100 \%$ & $\sim 10 \%$ \\
\hline Total & $100 \%$ & $\mathrm{~N} / \mathrm{A}$ & $50 \%-68 \%$ \\
\hline
\end{tabular}

power provided by the amplifiers is wasted by the antenna system on the feeder that connects the antenna to the radio-frequency unit (typical cable attenuation at $2 \mathrm{GHz}$ is 0.1 to $0.5 \mathrm{~dB} / \mathrm{m}$, which yields a typical $2 \mathrm{~dB}$ to $3 \mathrm{~dB}$ loss in traditional base station sites).

Note that the power needed for digital processing, operated at both the base-band and radio-frequency units, accumulates as low as $10 \%$ of the overall base station consumption. Furthermore, according to Huawei's data (which do not account for voltage rectifiers), $93 \%$ of the base station energy is spent for the digital processing, transmission, and air conditioning, the remaining $7 \%$ being spent for network devices and control units co-located in the base station site. These data also confirm that digital processing, transport, and control operations consume a negligible amount of power in comparison with the power amplifiers stage within the radio-frequency unit. Overall, amplifiers consumption can represent $60 \%$ of the total power, and, considering electronic and transmission devices only (i.e., not considering air conditioning), power amplifiers are responsible for as much as $80 \%$ of the base station consumption.

Interestingly, a relevant fraction of the power consumption in a base station is due to the air conditioning system [2], and a non-negligible quote of energy $(\sim 10 \%)$ is wasted due to AC/DC conversion needed to feed the electronics present in the digital devices (i.e., the control, transport, base band, and radio-frequency units) [2]. The power wasted by auxiliary equipments, such as the air conditioning and the voltage rectifier, can be quantified in at least one forth of the overall base station power demand, but can grow up to more than one third of the total power consumption.

In summary, the main causes of power consumption, and hence pollution, are the power amplifiers in the radiofrequency unit and the air conditioning system. The former stronger affects the latter, since about half of the power used for control, transport, processing and transmission is dissipated as heat by the power amplifiers. Air conditioning consumption also depends on the environmental conditions and the design of the base station spaces, and it can be as power-hungry as the power amplifiers. Remarkably, newly proposed efficient power amplifiers might enable a $20 \%$ of economy with respect to DPD power amplifiers, and $40 \%$ with respect to traditional amplifiers. Furthermore, these new power amplifier allow to eliminate the need for AC, and allow to use renewable power sources, which do not require the presence of power rectifiers, and makes a total potential energy economy up to $50 \%$ and GHG emissions down to almost zero. Another $20 \%$ of economy can be obtained by optimizing the control and networking units of the base station.

Table 3 summarizes the data about operational costs that are available on the manufacturers' websites (e.g., published by Alcatel-Lucent, Ericsson, Huawei, Motorola, Nortel, Nokia Siemens Networks, SEI, Nujira, ZTE). The table shows that each base station component has a different impact on the overall operational costs. Control and transport units are considered together, since they are commonly deployed on a single physical device. Similarly, specific data about the stand-alone base-band unit are not available, while it is possible to obtain the overall consumption of the transmission units (base-band, radio-frequency, and antennas), the cost due to the power amplifiers, and the data about the antenna system. Hence we split these cost in three parts: $(i)$ the cumulative consumption due to digital processing, which includes all base-band costs plus the digital processing cost at the radio-frequency unit; ( $i i$ ) the remaining portion of radio-frequency consumption, i.e., the power amplifiers consumption, which is dissipated as heat; and $($ iii $)$ the consumption due to antennas. The table also includes costs due to air conditioning and voltage rectifiers $(\mathrm{AC} / \mathrm{DC})$.

Table 3 also enlightens how technological improvements might dramatically reduce operational costs up to nearly $70 \%$. The power save might be attained by making the electronic and network devices more efficient (more than $20 \%$ of gain can be obtained by considering the first three rows in the table), shorten the antenna feeder (another $10 \%$ reduction of overall consumption), removing the need for the air conditioning system by reducing the heat produced by the power amplifiers (up to $25 \%$ less power), and eliminate the need for voltage rectifiers by using DC generators, 
e.g., renewable power supplies based on solar panels that might save an extra 10\%. Note that efficient electronics alone enable as much saving as $50 \%$ or more of the overall consumption. In fact, efficient electronics are necessary for designing small boards that can be located close to the antenna and do not require air conditioning.

\subsection{Measurements on base station consumption}

There are quite a few data available in the scientific literature about the power consumption of each component of a base station, either for $2 \mathrm{G}$ base transceiver stations or $3 \mathrm{G}$ Nodes B, which is how $3 \mathrm{G}$ base stations are referred to. In contrast, many base station vendors advertise the average power consumption of their equipments, and usually provide aggregate data, i.e., per base station, or even per network consumption data.

In [2], the authors report on a measurement study about a typical $2 \mathrm{G} / 3 \mathrm{G}$ base station in the Vodafone Portuguese mobile network. The experimental study shows that for a common outdoor base station, operating three radiofrequency units (one for GSM in the $900 \mathrm{MHz}$ band, one for GSM in $1800 \mathrm{MHz}$ band, and one for UMTS), about 76\% of the power consumption is due to the electronics, while about $24 \%$ of the total power is consumed by the cooling system. The same study shows that the cost of the GSM radio-frequency unit is roughly $30 \%$ higher than the cost of the UMTS radio-frequency unit.

Noticeably, for both technologies, the power consumption only slightly fluctuates over time (cf. Figure 22 in [2]). GSM consumption, in a time window of 15 minutes, is reported to be 0.27 to $0.30 \mathrm{kWh}$, i.e., it exhibits an average power consumption ranging from $1.08 \mathrm{~kW}$ in the off-peak hours to $1.20 \mathrm{~kW}$ during the peak hours. On the other hand, the UMTS consumption, in a time window of 15 minutes, ranges from $0.19 \mathrm{kWh}$ to $0.22 \mathrm{kWh}$, and correspondingly the average drained power approximately ranges from $0.76 \mathrm{~kW}$ to $0.88 \mathrm{~kW}$ during a day. Note also that radio-frequency units are DC-operated. With the efficiency of the AC/DC rectifier used at the tested base station being about $92 \%$, the actual power consumption due to each radio-frequency unit is actually $8.7 \%$ higher than that discussed before (cf. Section IV in [2]).

The measurements reported in [2] suggest that the presence of traffic only slightly affects the power consumption at the base station. A major reduction in the power consumption is then possible only by turning off the base station $\operatorname{radio}(\mathrm{s})$.

\section{Conclusions}

Throughout the paper, we have surveyed the strategies adopted by base station manufacturers and operators on the road towards a low-cost and environment-friendly wireless networking. Most of the current green best practices concern the rationalization of $(i)$ capital expenditures, by optimizing the base station site architecture and the distribution of the sites over the targeted coverage area, and $(i i)$ operational expenditures, by minimizing the energy consumption of electronic devices and reducing the need for cooling systems. In addition, since the majority of the operational expenditures are due to the electricity consumption of radio-frequency transceivers, new software-based management tools have been coming into play, which try to enforce a sleep mode on those equipments that are expected to handle low or no traffic during the off-peak hours.

Based on the data available on the per-device energy consumption, the survey strongly suggests that high efficiency electronics might dramatically reduce the consumption of next generation OFDMA-based systems. However, a predominant portion of the base station costs is incurred as soon as the radio devices are turned on, and it does not depend on the traffic flowing through the devices. Thereby, smart and flexible sleep mechanisms should be provided in order to make the energy consumption depend on the time-varying traffic load.

\section{References}

[1] E. Dahlman, S. Parkvall, J. Skold, P. Beming, 3G Evolution: HSPA and LTE for Mobile Broadband, Second Edition, Academic Press, Oxford, UK, 2008.

[2] F. Corrêa Alegria, F.A. Martins Travassos, Implementation details of an automatic monitoring system used on a Vodafone radiocommunication base station, IAENG Engineering Letters 16 (4).

[3] S. Alouf, E. Altman, A.P. Azad, Analysis of an M/G/1 queue with repeated inhomogeneous vacations with application to IEEE 802.16e power saving mechanism, in: Proc. of QEST, Saint-Malo, France, 2008, pp. 27-36. 
[4] A.P. Azad, S. Alouf, E. Altman, V. Borkar, G. Paschos, Optimal sampling for state change detection with application to the control of sleep mode, in: Proc. of 48th IEEE Conference on Decision and Control (CDC), Shanghai, China, 2009.

[5] L. Saker, S.-E. Elayoubi, H.O. Scheck, Energy-aware system selection in cooperative networks, in: Proc. of VTC Fall, 2009.

[6] 3GPP TS 25.214, Physical layer procedures (FDD), release 8.

[7] S. Shepherd, J. Orriss, S. Barton, Asymptotic limits in peak envelope power reduction by redundant coding in orthogonal frequency-division multiplex modulation, IEEE Transactions on Communications 46 (1) (1998) 5-10.

[8] S.-C. Jung, O. Hammi, F.M.G. Ghannouchi, Design optimization and DPD linearization of GaN-based unsymmetrical Doherty power amplifiers for 3G multicarrier applications, IEEE Transaction on Microwave Theory and Techniques 57 (9) (2009) 2105-2113.

[9] W.H. Doherty, A new high efficiency power amplifier for modulated wave, in: Proc. of IRE, Vol. 24, 1936, pp. $1163-1182$.

[10] Y.-S. Lee, M.-W. Lee, S.-H. Kam, Y.-H. Jeong, Highly linear and efficient asymmetrical Doherty power amplifiers with adaptively bias-controlled predistortion drivers, in: Proc. of IEEE MTT-S International Microwave Symposium Digest, 2009, pp. 1393-1396. 